\title{
UMA ABORDAGEM ANALÍTICA PARA DETECÇÃO DE PONTOS LIMITES E DE BIFURCAÇÃO
}

\section{An analytical approach for the detection of limit points and bifurcation points in structural systems}

\author{
William Taylor Matias Silva', María Paz Duque Gutiérrez²; Wellington Andrade da Silva
}

Recebido em 16 de fevereiro de 2016; recebido para revisão em 03 de junho de 2016; aceito em 19 de julho de 2016; disponível on-line em 15 de agosto de 2016.

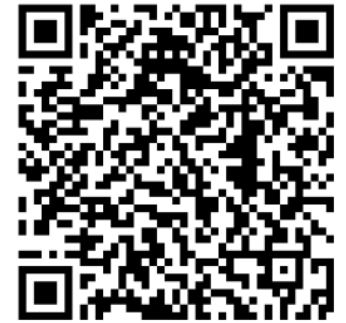

PALAVRAS CHAVE:

Descrição Lagrangiana total;

Análise não linear geométrica;

Pontos de bifurcação; Pontos limite;

Elementos de treliça plana.

\section{KEYWORDS:}

Total Lagrangian

formulation;

Geometrical nonlinearty;

Bifurcation points;

Limit points;

Plane truss element;

* Contato com os autores:

1e-mail: taylor@unb.br (W. T. M. Silva)

Professor Doutor do Departamento de Engenharia Civil e Ambiental da Universidade de Brasília - UnB.

2e-mail: mpazduque@gmail.com (M.P.D. Gutiérrez)

Doutoranda em Estruturas e Construção Civil pela Universidade de Brasília - UnB.

${ }^{3}$ e-mail: wellington_andrade@ufg.br (W. A. da Silva )

Professor Doutor da Universidade Federal de Goiás, Regional Catalão - UFG. lineares para projetar sistemas estruturais mais seguros. understanding is necessary for designing safer structural systems.

RESUMO: Neste trabalho descreve-se analiticamente de maneira detalhada a detecção e a classificação de pontos críticos na trajetória primária de equilíbrio de sistemas estruturais. Utiliza-se a Formulação Lagrangiana Total para descrever a cinemática de um elemento de barra biarticulado 2D. Através desta formulação obtém-se o vetor de forças internas e a matriz de rigidez tangente que levam em conta os efeitos da não linearidade geométrica. Assume-se um modelo constitutivo linear elástico para o estado uniaxial de tensão-deformação, usando a deformação de Green-Lagrange e a tensão axial do segundo tensor de Piola-Kirchhoff que são energeticamente conjugados. Como estudo de caso apresenta-se uma treliça plana hiperestática composta com 3 elementos biarticulados 2D e com dois graus de liberdade. Por fim, determinam-se as condições geométricas e físicas para a coalescência entre os pontos limites e de bifurcação. A principal contribuição deste trabalho é demonstrar a necessidade de compreender melhor os fenômenos não

ABSTRACT: Using an analytical this paper describes in detail the detection and classification of critical points in the primary equilibrium path of structural systems. The Total Lagrangian formulation is employed to describe the kinematics of a 2D bar element. With this formulation, the internal force vector and the tangent stiffness matrix including the geometric nonlinearity effects are obtained. An elastic linear constitutive model is assumed for the uniaxial stress-strain state. Such model uses the Green-Lagrange strain tensor and the second Piola-Kirchhoff axial stress tensor which are energetically conjugate tensors. As a study case, the article presents a statically inderminate plane truss discretized with three 2D bar elements. Finally, the geometrical and physical conditions for the coalescence between limit and bifurcation points are determined. The main contribution of this work is to demonstrate the need to better understanding the non linear phenomena. Such 


\section{INTRODUÇÃO}

Tornou-se quase imperativo, para o avanço da ciência, compreender e simular os fenômenos não lineares em diversas áreas do conhecimento, tais como, biomecânica, mecânica dos fluídos, geotécnica, mecânica dos sólidos, engenharia de tecidos humanos, etc. Por exemplo, nas indústrias aeronáutica, aeroespacial e petrolífera a análise não linear é imprescindível no projeto de diferentes tipologias estruturais aplicadas nesses setores. Nas últimas décadas muitos autores têm publicados livros texto abordando diferentes tópicos da análise não linear na área dos métodos numéricos aplicados a engenharia, por exemplo, recomenda-se a leitura das seguintes referências: Crisfield (1991), Crisfield (1997), Bathe (1996), Belytschko et al. (2000), Doyle (2001), Wriggers (2002), Borst et al (2012), Bonet e Wood (2008), Wriggers (2008), Krenk (2009). Decorre, daí a necessidade de difundir os conceitos básicos e fundamentais da análise não linear através do método dos elementos finitos, imbuído deste espírito, o trabalho aqui apresentado, sem maiores pretensões teóricas, tem como objetivo apresentar uma análise teórica e numérica da instabilidade estrutural que consiste na detecção e classificação de pontos singulares na trajetória de equilíbrio primária. Propõe-se utilizar elementos de barra biarticulados por sua simplicidade teórica, o que permite descrever, facilmente, a cinemática do movimento do elemento e obter o vetor de forças internas e a matriz de rigidez tangente analiticamente, que são elementos imprescindíveis para uma análise não linear em mecânica dos sólidos. Para simular deformações elásticas finitas, assume-se um modelo constitutivo hiperelástico para o estado uniaxial de tensão-deformação, utilizando a tensão axial do segundo tensor de PiolaKirchhoff e a deformação de Green-Lagrange, que formam um par energeticamente conjugado. Nos itens 2,3 e 4 descrevem-se a cinemática do elemento biarticulado 2D adotando a Formulação Lagrangiana Total (FLT), a obtenção do vetor de forças internas e a dedução da matriz de rigidez tangente, respectivamente. No item 5 faz-se uma abordagem analítica detalhada da detecção e classificação dos pontos singulares presentes na trajetória primária de equilíbrio de uma treliça plana hiperestática discretizada por três barras biarticuladas 2D. Por último, apresentam-se as conclusões finais e as referências bibliográficas utilizadas neste trabalho.

\section{DESCRIÇÃO CINEMÁTICA}

Seja um sistema de coordenadas globais cuja base é ortonormal conforme mostra-se na Figura 1. Para expressar as variáveis cinemáticas na configuração indeformada utilizam-se as coordenadas materiais $(X, Y)$, enquanto que, na configuração deformada as coordenadas espaciais $(x, y)$. Na configuração indeformada as coordenadas nodais do elemento biarticulado 2D são dadas por $\boldsymbol{X}_{A}=\left(X_{A}, Y_{A}\right)$ e $\boldsymbol{X}_{B}=\left(X_{B}, Y_{B}\right)$, respectivamente. Sua posição e comprimento iniciais são dados pela Equação 1.
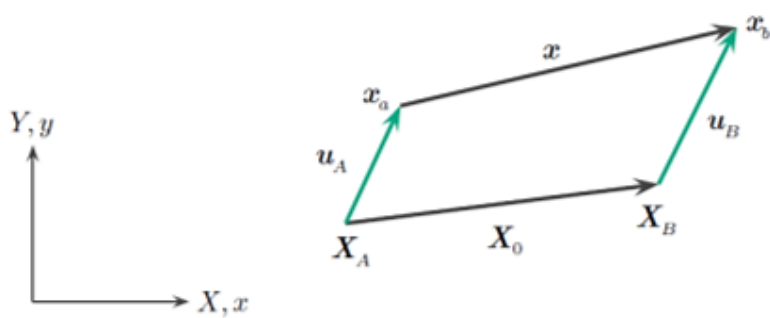

FIGURA 1: Movimento do elemento biarticulado 2D. FONTE: Autoria Própria (2016).

$$
\begin{gathered}
\overrightarrow{A B}=\boldsymbol{X}_{0}=\boldsymbol{X}_{B}-\boldsymbol{X}_{A} \\
l_{0}{ }^{2}=\boldsymbol{X}_{0}{ }^{T} \boldsymbol{X}_{0}
\end{gathered}
$$

Em que:

$\overrightarrow{A B}$ ou $\boldsymbol{X}_{0}$ : vetor posição na configuração indeformada.

$l_{0}$ : comprimento do elemento biarticulado $2 \mathrm{D}$ na configuração indeformada.

Enquanto que na configuração deformada as coordenadas nodais do elemento biarticulado $2 \mathrm{D}$ são dadas por $\boldsymbol{x}_{A}=\left(x_{A}, y_{A}\right)$ e $\boldsymbol{x}_{B}=\left(x_{B}, y_{B}\right)$, respectivamente. Conforme mostra-se na Figura 1 a coordenada atual do nó $A$ é dada por $\boldsymbol{x}_{A}=\boldsymbol{X}_{A}+$ $\boldsymbol{u}_{A}$, enquanto que a coordenada atual do nó $B$ se expressa como $\boldsymbol{x}_{B}=\boldsymbol{X}_{B}+\boldsymbol{u}_{B}$. Sendo que $\boldsymbol{u}_{A}=\left(u_{A}, v_{A}\right)$ é o deslocamento do nó $A \mathrm{e}$ 
$\boldsymbol{u}_{B}=\left(u_{B}, v_{B}\right)$ é o deslocamento do nó $B$. Portanto, a posição atual do elemento é dada por meio da Equação 2.

$$
\begin{gathered}
\overrightarrow{a b}=\boldsymbol{x}=\boldsymbol{x}_{B}-\boldsymbol{x}_{A} \\
\overrightarrow{a b}=\boldsymbol{x}=\left(\boldsymbol{X}_{B}+\boldsymbol{u}_{B}\right)-\left(\boldsymbol{X}_{A}+\boldsymbol{u}_{A}\right) \\
\overrightarrow{a b}=\boldsymbol{x}=\left(\boldsymbol{X}_{B}-\boldsymbol{X}_{A}\right)+\left(\boldsymbol{u}_{B}-\boldsymbol{u}_{A}\right) \\
\overrightarrow{a b}=\boldsymbol{x}=\boldsymbol{X}_{0}+\boldsymbol{u}_{B A}
\end{gathered}
$$

Em que:

$\overrightarrow{a b}$ ou $\boldsymbol{x}$ : vetor posição na configuração deformada.

Sendo que as coordenadas do vetor posição atuais são dadas por $x_{B A}=x_{B}-x_{A}$ e $y_{B A}=y_{B}-y_{A}$, respectivamente. $\boldsymbol{u}_{B A}=\boldsymbol{u}_{B}-\boldsymbol{u}_{A}$ é o vetor de deslocamentos nodais relativos conforme mostra-se na Figura 2. Por outro lado, o comprimento atual se expressa por meio da Equação 3.

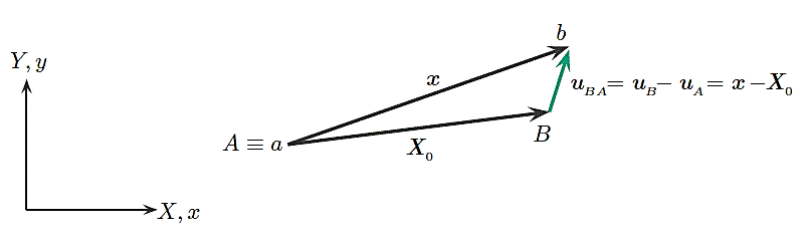

FIGURA 2: Vetor de deslocamentos nodais relativos. FONTE: Autoria Própria (2016).

$$
l^{2}=\boldsymbol{x}^{T} \boldsymbol{x}=\left(\boldsymbol{X}_{0}+\boldsymbol{u}_{B A}\right)^{T}\left(\boldsymbol{X}_{0}+\boldsymbol{u}_{B A}\right)
$$

Neste trabalho adota-se a formulação Lagrangiana Total para descrever o movimento do elemento biarticulado 2D, portanto serão usadas as coordenadas materiais $(X, Y)$ e a configuração indeformada para definir a medida de deformação do elemento. Dentre algumas famílias de deformação descritas em coordenadas materiais existentes na literatura técnica, adota-se neste trabalho, a medida de deformação de GreenLagrange que compara os quadrados dos comprimentos atual $l$ e inicial $l_{0}$ do elemento é dada por meio da Equação 4.

$$
\varepsilon_{G}=\frac{l^{2}-l_{0}{ }^{2}}{2 l_{0}{ }^{2}}=\frac{1}{l_{0}{ }^{2}}\left(\boldsymbol{X}_{0}{ }^{T} \boldsymbol{u}_{B A}+\frac{1}{2} \boldsymbol{u}_{B A}{ }^{T} \boldsymbol{u}_{B A}\right)
$$

Em que:

$\varepsilon_{G}$ : Deformação de Green-Lagrange.

Note-se que esta medida de deformação possui termos quadráticos em relação aos deslocamentos nodais relativos.
Para obter o vetor de forças internas utiliza-se o Princípio dos Trabalhos Virtuais (PTV), portanto é necessário aplicar uma variação virtual no campo de deslocamentos na configuração de equilíbrio atual, conforme mostra-se na Figura 3, o que implica em uma variação virtual da deformação de Green-Lagrange que se escreve conforme a Equação 5.

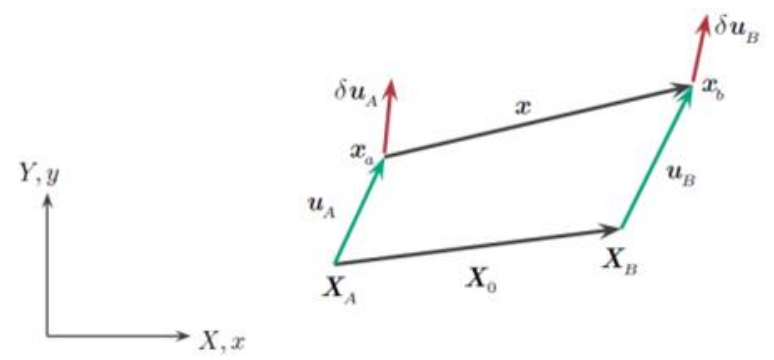

FIGURA 3:Variação virtual dos deslocamentos nodais. FONTE: Autoria Própria (2016).

$$
\begin{gathered}
\delta \varepsilon_{G}=\frac{1}{l_{0}^{2}}\left(\boldsymbol{X}_{0}^{T} \delta \boldsymbol{u}_{B A}+\frac{1}{2} \delta \boldsymbol{u}_{B A}^{T} \boldsymbol{u}_{B A}\right. \\
\left.+\frac{1}{2} \boldsymbol{u}_{B A}{ }^{T} \delta \boldsymbol{u}_{B A}\right) \\
\delta \varepsilon_{G}=\frac{1}{l_{0}^{2}}\left(\boldsymbol{X}_{0}^{T} \delta \boldsymbol{u}_{B A}+\boldsymbol{u}_{B A}{ }^{T} \delta \boldsymbol{u}_{B A}\right) \\
\delta \varepsilon_{G}=\frac{1}{l_{0}^{2}}\left(\boldsymbol{X}_{0}+\boldsymbol{u}_{B A}\right)^{T} \delta \boldsymbol{u}_{B A}=\frac{1}{l_{0}^{2}} \boldsymbol{x}^{T} \delta \boldsymbol{u}_{B A}
\end{gathered}
$$

\section{Em que:}

$\delta \varepsilon_{G}$ : Variação virtual da deformação de GreenLagrange.

Sendo que $\delta \boldsymbol{u}_{B A}=\delta \boldsymbol{u}_{B}-\delta \boldsymbol{u}_{A}$ é a variação virtual do vetor de deslocamentos relativos. Note-se que a variação virtual da deformação de Green-Lagrange consiste na projeção da variação virtual do vetor dos deslocamentos relativos sobre a posição atual do elemento definida pelo vetor $\boldsymbol{x}$ e escalado por $l_{0}{ }^{2}$.

\section{VETOR DE FORÇAS NODAIS}

Como mostra-se na Figura 4, seja $f_{A}=\left(f_{A_{x}}, f_{A_{y}}\right)$ o vetor de forças do nó $A$, e $f_{B}=\left(f_{B_{x}}, f_{B_{y}}\right)$ o vetor de forças do nó $B$, respectivamente. Para obter estes vetores de forças nodais aplica-se o PTV na configuração indeformada pois se está utilizando a medida de deformação de Green-Lagrange, desta maneira expressa-se este princípio pela Equação 6 . 

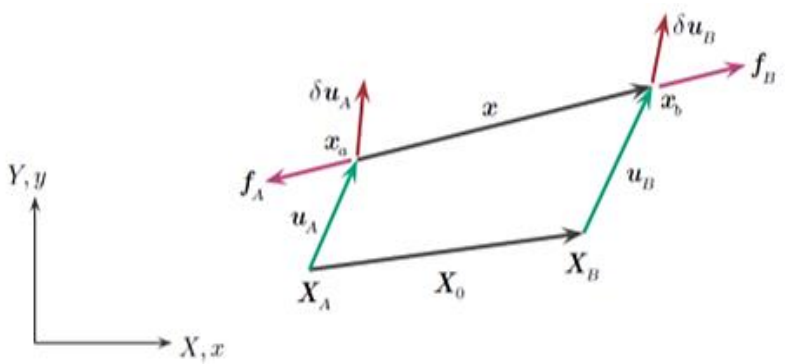

FIGURA 4: Vetor de forças nodais e a variação virtual dos deslocamentos nodais.

FONTE: Autoria Própria (2016)

$$
\delta V=\int_{0}^{l_{0}} N \delta \varepsilon_{G} d s-\boldsymbol{f}_{A}^{T} \delta \boldsymbol{u}_{A}-\boldsymbol{f}_{B}^{T} \delta \boldsymbol{u}_{B}=0
$$

Sendo que $N$ é o esforço axial que atua no elemento e é dado por $N=\sigma_{G} A$. Lembrando que a tensão axial $\sigma_{G}$ é energeticamente conjugada com a medida de deformação de Green-Lagrange e é uma das tensões normais do segundo tensor de tensões de Piola-Kirchoff. Substituindo a Equação $5 c$ na Equação 6, tem-se que (Equação 7).

$$
\begin{aligned}
& \delta V=\int_{0}^{l_{0}} \frac{N}{l_{0}{ }^{2}} \boldsymbol{x}^{T} \delta \boldsymbol{u}_{B A} d s-\boldsymbol{f}_{A}{ }^{T} \delta \boldsymbol{u}_{A}-\boldsymbol{f}_{B}{ }^{T} \delta \boldsymbol{u}_{B}=0 \\
& \begin{array}{c}
\delta V=\int_{0}^{l_{0}} \frac{N}{l_{0}^{2}} \boldsymbol{x}^{T}\left(\delta \boldsymbol{u}_{B}-\delta \boldsymbol{u}_{A}\right) d s-\boldsymbol{f}_{A}{ }^{T} \delta \boldsymbol{u}_{A}-\boldsymbol{f}_{B}{ }^{T} \delta \boldsymbol{u}_{B} \\
=0
\end{array} \\
& \delta V=\delta \boldsymbol{u}_{A}^{T}\left(-\int_{0}^{l_{0}} \frac{N}{l_{0}^{2}} \boldsymbol{x} d s-\boldsymbol{f}_{A}\right) \\
& +\delta \boldsymbol{u}_{B}^{T}\left(-\int_{0}^{l_{0}} \frac{N}{l_{0}^{2}} \boldsymbol{x} d s-\boldsymbol{f}_{B}\right)=0 \\
& \boldsymbol{f}_{A}=-\frac{N}{l_{0}} \boldsymbol{x} ; \boldsymbol{f}_{B}=\frac{N}{l_{0}} \boldsymbol{x}
\end{aligned}
$$

Conforme mostra-se na Figura 4, por convenção, o sinal do vetor de forças internas $\boldsymbol{f}_{A}$ é negativo porque está no sentido contrario do vetor posição $\boldsymbol{x}$, enquanto que o sinal do vetor de forças internas $\boldsymbol{f}_{B}$ é positivo porque possui o mesmo sentido do vetor $\boldsymbol{x}$. Neste trabalho assume-se que $\sigma_{G}=E \varepsilon_{G}$, onde $E$ é o módulo de elasticidade longitudinal do material, assim o esforço axial pode ser definido como $N=E A \varepsilon_{G}$ e a Equação $7 d$ pode ser reescrita conforme a Equação 8.

$$
\begin{aligned}
& \boldsymbol{f}_{A}=-\frac{E A}{l_{0}} \varepsilon_{G} \boldsymbol{x} ; \quad \boldsymbol{f}_{B}=\frac{E A}{l_{0}} \varepsilon_{G} \boldsymbol{x} \\
& \boldsymbol{f}_{A}=-E A \varepsilon_{G}\left\{\begin{array}{c}
\frac{x_{B A}}{l_{0}} \\
\frac{y_{B A}}{l_{0}}
\end{array}\right\} ; \boldsymbol{f}_{B}=E A \varepsilon_{G}\left\{\begin{array}{c}
\frac{x_{B A}}{l_{0}} \\
\frac{y_{B A}}{l_{0}}
\end{array}\right\}
\end{aligned}
$$

\section{MATRIZ DE RIGIDEZ TANGENTE}

Ao aplicar um incremento infinitesimal nos vetores de deslocamentos nodais $\boldsymbol{u}_{A}$ e $\boldsymbol{u}_{B}$ na configuração deformada, obtém-se um incremento infinitesimal dos vetores de forças internas $\boldsymbol{f}_{A}$ e $\boldsymbol{f}_{B}$, respectivamente, através da matriz de rigidez tangente. Portanto, define-se a relação entre os incrementos infinitesimais dos vetores de forças internas e dos vetores de deslocamentos nodais conforme a Equação 9.

$$
\left\{\begin{array}{l}
d f_{A} \\
d f_{B}
\end{array}\right\}=K_{T}\left\{\begin{array}{l}
d u_{A} \\
d u_{B}
\end{array}\right\}
$$

Sendo que $\boldsymbol{K}_{T}$ é a matriz de rigidez tangente de ordem $4 \times 4$. Portanto, levando em conta a Equação (2d) e diferenciando-se a Equação 7d em relação ao vetor de deslocamentos relativos, obtém-se que (Equação 10)

$$
\begin{gathered}
d \boldsymbol{q}_{A}=-\boldsymbol{x} \frac{d N}{l_{0}}-\frac{N}{l_{0}} d \boldsymbol{x} \\
d \boldsymbol{q}_{A}=-\left(\frac{\boldsymbol{x}}{l_{0}} \frac{d N}{d \boldsymbol{u}_{B A}}+\frac{N}{l_{0}} \boldsymbol{I}\right) d\left(\boldsymbol{u}_{B}-\boldsymbol{u}_{A}\right) \quad \text { Eq.[10] } \\
d \boldsymbol{q}_{B}=-d \boldsymbol{q}_{A}
\end{gathered}
$$

Sendo $I$ a matriz identidade de ordem $2 \times 2$. Por outro lado, diferenciando-se o esforço axial em relação ao vetor dos deslocamentos relativos, e levando em conta as Equações 2d, 4 e 5, chega-se a Equação 11.

$$
\begin{gathered}
\frac{d N}{d \boldsymbol{u}_{B A}}=E A \frac{d \varepsilon_{G}}{d \boldsymbol{u}_{B A}}=\frac{E A}{l_{0}{ }^{2}}\left(\boldsymbol{X}_{0}{ }^{T}+\boldsymbol{u}_{B A}{ }^{T}\right) \\
=\frac{E A}{l_{0}{ }^{2}} \boldsymbol{x}^{T}
\end{gathered}
$$

Por último, substituindo a Equação 11 na Equação 10b e levando em conta a Equação 10c, obtém-se que (Equação 12). 


$$
\begin{array}{r}
\left\{\begin{array}{l}
d \boldsymbol{q}_{A} \\
d \boldsymbol{q}_{B}
\end{array}\right\}=\left(\frac{E A}{l_{0}{ }^{3}}\left[\begin{array}{rr}
\boldsymbol{x} \otimes \boldsymbol{x} & -\boldsymbol{x} \otimes \boldsymbol{x} \\
-\boldsymbol{x} \otimes \boldsymbol{x} & \boldsymbol{x} \otimes \boldsymbol{x}
\end{array}\right]\right. \\
\left.+\frac{N}{l_{0}}\left[\begin{array}{rr}
\boldsymbol{I} & -\boldsymbol{I} \\
-\boldsymbol{I} & \boldsymbol{I}
\end{array}\right]\right)\left\{\begin{array}{l}
d \boldsymbol{u}_{A} \\
d \boldsymbol{u}_{B}
\end{array}\right\}
\end{array}
$$

Com (Equação 13)

$$
\begin{aligned}
& \boldsymbol{K}_{M}=\frac{E A}{l_{0}{ }^{3}}\left[\begin{array}{rr}
\boldsymbol{x} \otimes \boldsymbol{x} & -\boldsymbol{x} \otimes \boldsymbol{x} \\
-\boldsymbol{x} \otimes \boldsymbol{x} & \boldsymbol{x} \otimes \boldsymbol{x}
\end{array}\right] \\
& \boldsymbol{K}_{\sigma}=\frac{N}{l_{0}}\left[\begin{array}{rr}
\boldsymbol{I} & -\boldsymbol{I} \\
-\boldsymbol{I} & \boldsymbol{I}
\end{array}\right] \\
& \boldsymbol{x} \otimes \boldsymbol{x}=\left[\begin{array}{cc}
x_{B A}{ }^{2} & x_{B A} y_{B A} \\
x_{B A} y_{B A} & y_{B A}^{2}
\end{array}\right]
\end{aligned}
$$

Sendo $\boldsymbol{K}_{M}$ a matriz de rigidez material, de ordem $4 \times 4$, que depende do vetor posição atual do elemento $x$ cujas componentes são: $x_{B A}=x_{B}-x_{A}$ e $y_{B A}=y_{B}-y_{A} \cdot \boldsymbol{K}_{\sigma}$ é a matriz de rigidez geométrica que depende do esforço axial $N$ e é de ordem $4 x 4$ e o símbolo $\otimes$ é o produto tensorial ou aberto. Portanto, a matriz de rigidez tangente se expressa como $\boldsymbol{K}_{T}=\boldsymbol{K}_{M}+\boldsymbol{K}_{\sigma}$.

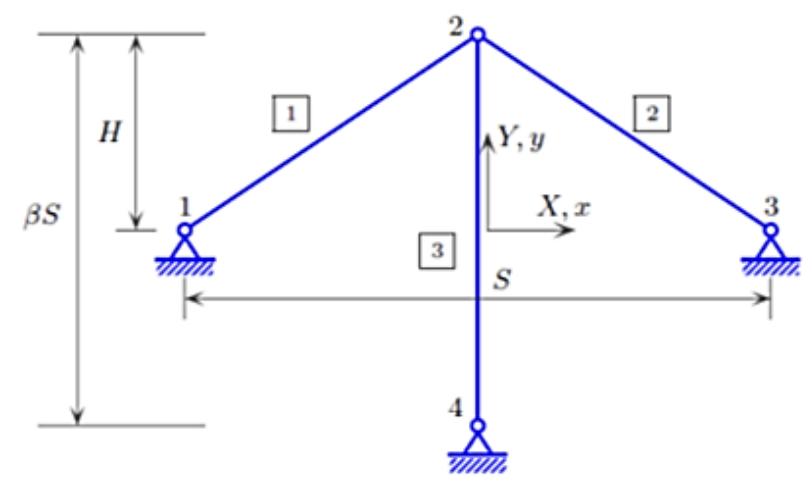

[a]

\section{FORMULAÇÃO ANALÍTICA}

Seja uma treliça plana com um grau de indeterminação estática conforme mostra-se na Figura 5a. Nesta figura mostram-se as propriedades geométricas e as condições de contorno, onde $H$ é a altura e $S$ é o vão. Considera-se que a rigidez axial de todas as barras da treliça é igual a $E A_{0}$, onde $E$ é o módulo de elasticidade longitudinal e $A_{0}$ é área da seção transversal de cada barra indeformada. Discretiza-se a treliça com 3 elementos de barras biarticulado 2D conforme-se mostra-se na Figura $5 a$. O comprimento do terceiro elemento é $\beta S$, onde $\beta$ é um parâmetro adimensional. O nó 2 está livre para deslocar-se nas direções dos eixos $x$ e $y$, respectivamente. Os demais nós estão restringidos por apoios de segundo gênero. Na Tabela 1 detalham-se as conectividades adotadas para cada elemento da treliça.

TABELA 1: Conectividades dos elementos.

\begin{tabular}{ccc} 
Elemento & Nó inicial & Nó final \\
\hline 1 & 1 & 2 \\
2 & 2 & 3 \\
3 & 2 & 4 \\
\hline
\end{tabular}

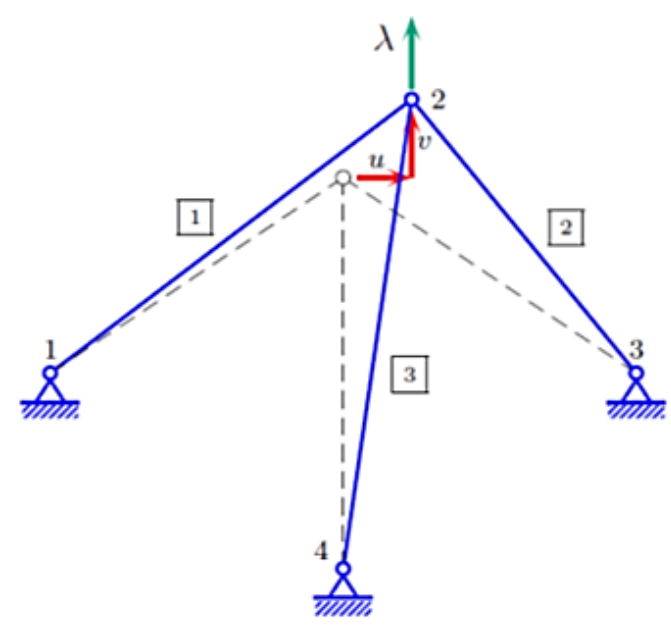

[b]

FIGURA 5: Treliça plana hiperestática, sendo: [a] configuração indeformada e [b] configuração deformada. FONTE: Autoria Própria (2016). 
Neste item faz-se a análise da estabilidade do equilíbrio da treliça plana hiperestática na configuração deformada como mostra-se na Figura 5b ao aplicar-se uma carga $\lambda$ na direção do eixo $y$. Estudam-se duas trajetórias de equilíbrio possíveis para este caso. A primeira condição refere-se à trajetória primária de equilíbrio onde o nó 2 irá deslocar-se somente na direção do eixo $y$ devido à simetria geométrica e das propriedades mecânicas da treliça, além de não haver carga aplicada na direção do eixo $x$ no nó 2 . Considera-se tanto o fator de carga aplicado quanto o deslocamento vertical no sentido negativo do eixo $y$. Neste caminho de equilíbrio a treliça irá apresentar um fenômeno de instabilidade conhecido na literatura técnica de snap-through que é a perda da capacidade portante da treliça quando a matriz de rigidez tangente da estrutura torna-se singular para um valor máximo de carga denominado de ponto limite. Na segunda condição de equilíbrio ocorre a flambagem da treliça no plano $(x, y)$ ainda que a carga esteja aplicada na direção do eixo $y$, o nó 2 irá deslocar-se na direção do eixo $x$, podendo ser no sentido positivo ou negativo deste eixo. Neste caso denomina-se caminho de equilíbrio secundário. Posteriormente, demonstra-se que estas trajetórias de equilíbrio interceptam-se em determinados pontos denominados de pontos de bifurcação.

Ao impor o campo de deslocamento $(u, v)$ no nó 2 da treliça, conforme mostra-se na Figura 5b, as barras irão deslocar-se, alterar seus comprimentos e direções, sofrer deformações e estarem submetidas a esforços axiais. Na Tabela 2 mostra-se a descrição cinemática, apresentada no item 2, de cada barra de maneira detalhada.

Para obter as trajetórias de equilíbrio primária e secundária é necessário montar o vetor de forças internas da treliça. Como somente o nó 2 pode-se deslocar, então basta montar o vetor de forças internas correspondente a este nó. Portanto levando em conta a Equação 8 monta-se o vetor de forças internas do nó 2 com a contribuição dos esforços internos de cada barra da treliça conectada a este nó como (Equação 14).

\section{TABELA 2: Descrição cinemática dos elementos.}

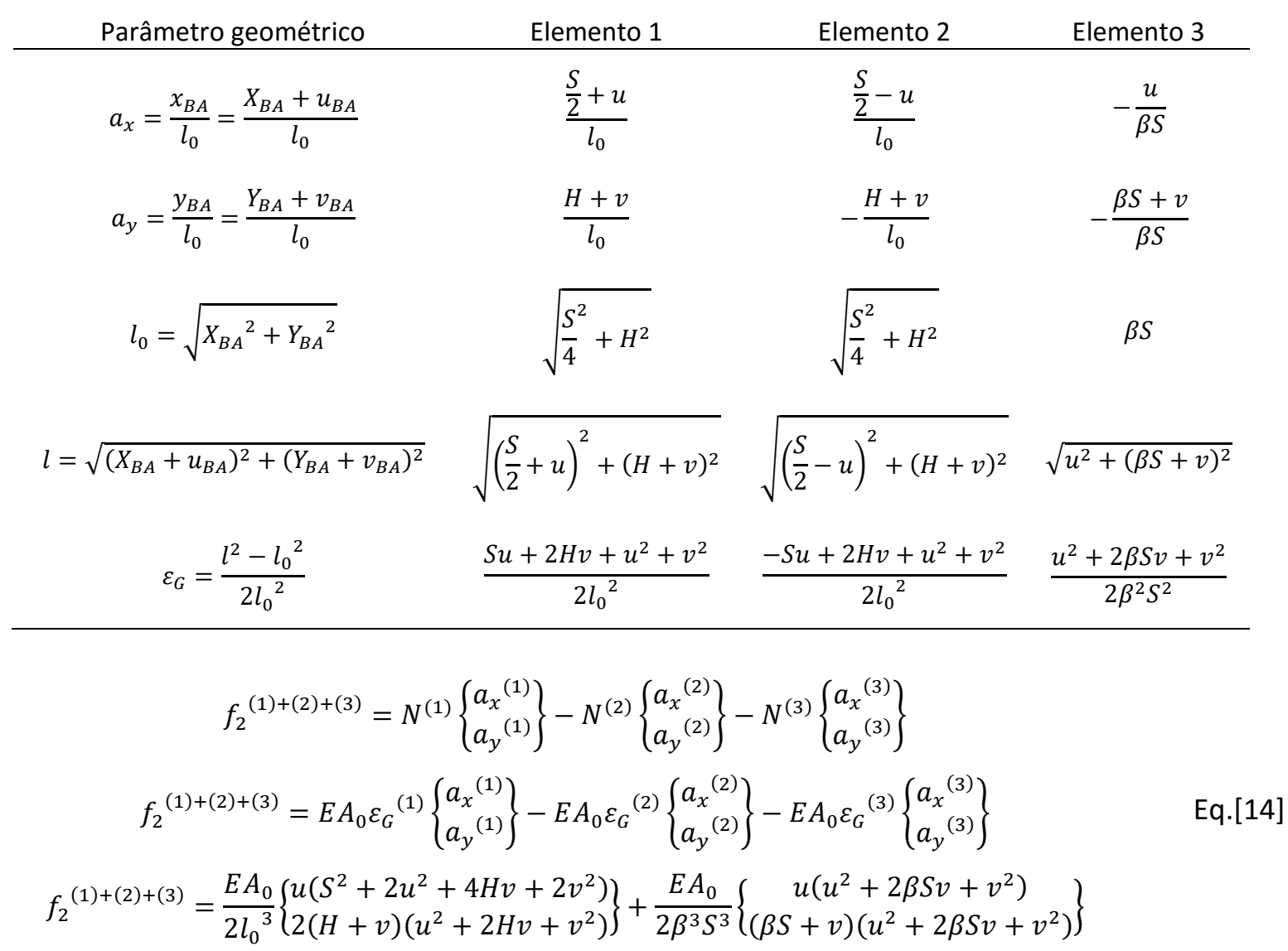


Note-se que o sinal negativo nos dois últimos termos da Equação 14a é devido ao nó 2 ser o nó inicial das barras 2 e 3, respectivamente. Por outro lado, na equação 14c foram utilizados os valores das deformações de cada barra dados na Tabela 2. Para que o nó 2 esteja em equilíbrio é necessário que o vetor de forças internas seja igual ao vetor de forças externas aplicadas neste nó, portanto obtém-se um sistema de duas equações não lineares que se expressam como (Equação 15).

\subsection{TRAJETÓRIA PRIMÁRIA DE EQUILÍBRIO}

Para obter a trajetória primária de equilíbrio impõe-se que o deslocamento horizontal do nó 2 seja igual a zero, $u=0$, no sistema de equações acima. Portanto, a primeira equação se anula e a segunda equação pode ser reescrita como a Equação 16.

Trata-se de um polinômio de grau três em função dos deslocamentos verticais do nó 2 da treliça. Para obter o gráfico desta curva adotaram-se os seguintes valores para as propriedades geométricas e mecânicas da treliça: $E=1, A_{0}=1, S=2$ e $H=3$. Atribui-se a $\beta$ os valores 1.0, 1.5 e 2.0. Desta maneira, obtém-se o gráfico de três trajetórias primárias de equilíbrio como mostra-se na Figura 6.

Nota-se que essas curvas possuem dois pontos extremos conhecidos como pontos limites. Nos próximos itens discute-se em detalhes a detecção e obtenção desses pontos.

\subsection{TRAJETÓRIA SECUNDÁRIA DE EQUILÍBRIO}

Inicialmente a treliça permanece na trajetória de equilíbrio primária, mas para determinadas condições geométricas e mecânicas, poderá flambar o que implica no deslocamento horizontal do nó 2. Para determinar essas condições imponhe-se no sistema de Equações 15 que $u \neq 0$. Como consequência, a primeira equação daquele sistema deve cumprir que (Equação 17).

Colocando em evidência o deslocamento $u$ na equação acima obtém-se que (Equação 18).

$$
\begin{gathered}
\frac{E A_{0}}{2 l_{0}^{3} \beta^{3} S^{3}}\left\{\begin{array}{c}
u\left(S^{2}+2 u^{2}+4 H v+2 v^{2}\right) \beta^{3} S^{3}+u\left(u^{2}+2 \beta S v+v^{2}\right) l_{0}{ }^{3} \\
2(H+v)\left(u^{2}+2 H v+v^{2}\right) \beta^{3} S^{3}+(\beta S+v)\left(u^{2}+2 \beta S v+v^{2}\right) l_{0}{ }^{3}
\end{array}\right\}=\left\{\begin{array}{l}
0 \\
\lambda
\end{array}\right\} \\
\lambda^{p}=\frac{E A_{0}}{2 l_{0}^{3} \beta^{3} S^{3}}\left(\left(4 H^{2} \beta^{3} S^{3}+2 \beta^{2} S^{2} l_{0}{ }^{3}\right) v+\left(6 H \beta^{3} S^{3}+3 \beta S l_{0}^{3}\right) v^{2}+\left(2 \beta^{3} S^{3}+l_{0}^{3}\right) v^{3}\right)
\end{gathered}
$$

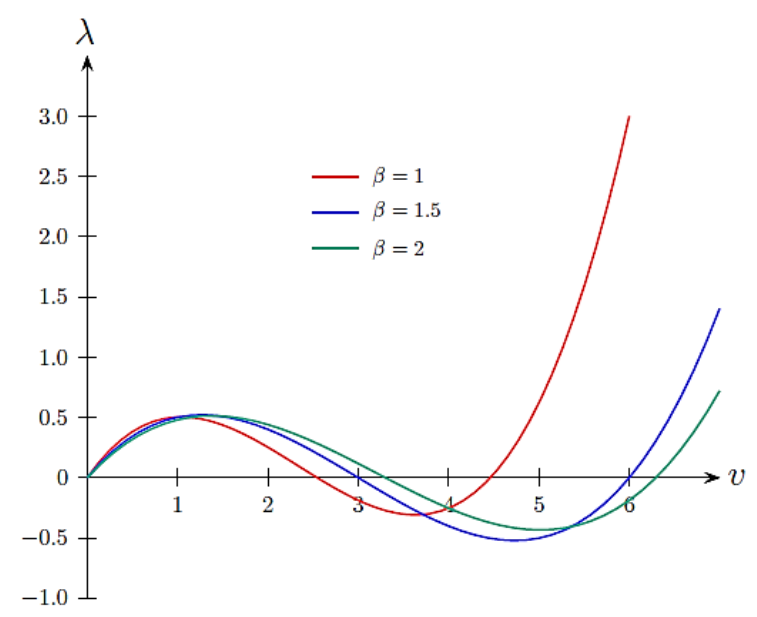

FIGURA 6: Trajetórias primárias de equilíbrio. FONTE: Autoria Própria (2016).

$$
\begin{gathered}
\left(S^{2}+2 u^{2}+4 H v+2 v^{2}\right) \beta^{3} S^{3}+\left(u^{2}+2 \beta S v+v^{2}\right) l_{0}^{3}=0 \\
u= \pm \sqrt{-v^{2}-\frac{\left(4 H \beta^{3} S^{3}+2 \beta S l_{0}^{3}\right)}{\left(2 \beta^{3} S^{3}+l_{0}^{3}\right)} v-\frac{\beta^{3} S^{5}}{\left(2 \beta^{3} S^{3}+l_{0}^{3}\right)}}
\end{gathered}
$$


Que é válida para a seguinte restrição (Equação 19).

$$
\begin{gathered}
v^{2}+\frac{\left(4 H \beta^{3} S^{3}+2 \beta S l_{0}^{3}\right)}{\left(2 \beta^{3} S^{3}+l_{0}^{3}\right)} v+\frac{\beta^{3} S^{5}}{\left(2 \beta^{3} S^{3}+l_{0}^{3}\right)}<0 \\
\frac{-\beta S\left(2 H \beta^{2} S^{2}+l_{0}^{3}\right)}{\left(2 \beta^{3} S^{3}+l_{0}^{3}\right)}-\Delta<v \\
v<\frac{-\beta S\left(2 H \beta^{2} S^{2}+l_{0}^{3}\right)}{\left(2 \beta^{3} S^{3}+l_{0}^{3}\right)}+\Delta \\
\Delta=\beta S \sqrt{\frac{\left(2 H \beta^{2} S^{2}+l_{0}^{3}\right)^{2}-\beta S^{3}\left(2 \beta^{3} S^{3}+l_{0}^{3}\right)}{\left(2 \beta^{3} S^{3}+l_{0}^{3}\right)}} \\
\Delta^{*}=\left(2 H \beta^{2} S^{2}+l_{0}^{3}\right)^{2}-\beta S^{3}\left(2 \beta^{3} S^{3}+l_{0}^{3}\right)>0
\end{gathered}
$$

Mostra-se na Figura 7 a relação entre os deslocamentos vertical e horizontal do nó 2 quando ocorre a flambagem da treliça dada pela Equação 18. Em todas as simulações a seguir assumem-se os seguintes dados para a treliça mostrada na Figura 5: $\left(E=1, A_{0}=1, S=2 H=3, \beta=1, \beta=1.5, \beta=2\right)$. Observa-se nesta figura que valores crescentes de $\beta$ implica em valores crescentes de $u$ e $v$, pois aumenta-se o comprimento da barra vertical $3 \mathrm{da}$ treliça e diminui-se sua rigidez axial, o que confere uma maior flexibilidade ao deslocamento do nó 2 . Nota-se na Figura 7 que inicialmente 0 deslocamento $u$ é nulo, depois ocorre a bifurcação para a trajetória secundária quando o valor de $u$ é crescente até um certo valor máximo para em seguida decrescer até zero quando a treliça retorna à trajetória primária. Observa-se nesta figura que do ponto de vista matemático o deslocamento $u$ pode ser no sentido positivo ou negativo do eixo $x$. A segunda restrição que surge para que ocorra a flambagem é que o discriminante da Equação 19d seja maior que zero. Note-se que essa restrição é essencialmente geométrica, portanto a ocorrência ou não da flambagem depende somente das características geométricas da treliça. Na Figura 8 mostra-se a partir de que valores de $H$, para cada valor de $\beta$, ocorre a bifurcação. Por fim, ao substituir a Equação 18 na Equação 16 obtém-se a trajetória secundária de equilíbrio em função do deslocamento vertical que se escreve conforme a Equação 20.

$$
\begin{aligned}
\lambda^{s}=\frac{E A_{0}}{2 l_{0}{ }^{3}\left(2 \beta^{3} S^{3}+\right.} & \left.l_{0}^{3}\right) \\
+ & {\left[\left(H^{2}+4 \beta^{2} S^{2}\right) l_{0}{ }^{3}\right.} \\
& \left.-2 \beta^{3} S^{5}\right) v \\
& \left.-\beta S^{3}\left(2 \beta^{2} S^{2} H+l_{0}{ }^{2}\right)\right]
\end{aligned}
$$

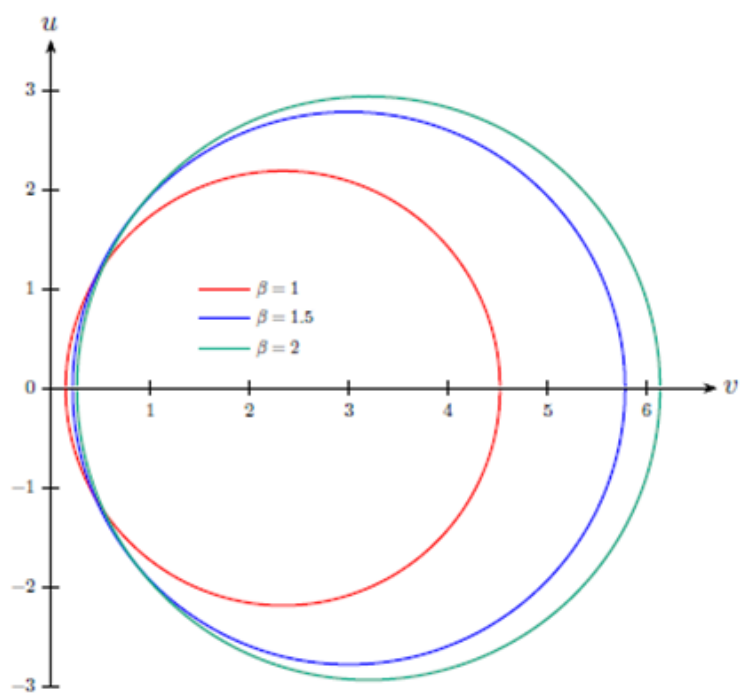

FIGURA 7: Projeção das trajetórias de equilíbrio secundárias no plano (u,v). FONTE: Autoria Própria (2016).

As trajetórias de equilíbrio dadas pelas Equações 16 e 20 estão em função do deslocamento vertical do nó 2 da treliça mostrada na Figura $5 a$. Nas Figuras 9a, 9b e 9c mostram-se as trajetórias de equilíbrio primária e secundária dadas pelas Equações 16 e 20, respectivamente, obtidas para os diferentes valores de $\beta$ adotados neste trabalho. Notam-se nessas figuras os pontos de bifurcação onde ocorre a intersecção entre as trajetórias primária e secundária de equilíbrio. Podem ser observados também, que os pontos limites que representam os extremos das trajetórias de equilíbrio primária. Na Figura 9d mostram-se as trajetórias de equilíbrio em função dos deslocamentos horizontal e vertical do nó 2, novamente, observa-se a intersecção entre as trajetórias primária e secundária de equilíbrio mostrada no plano $(\lambda, v, u)$. Observa-se na Figura 9d que a trajetória de equilíbrio dada pela Equação 20 é a projeção da trajetória de equilíbrio secundária no plano $(\lambda, v)$. Por outro lado, a projeção das trajetórias de equilíbrio secundárias mostradas nesta figura no plano $(v, u)$ é mostrada na Figura 7. 


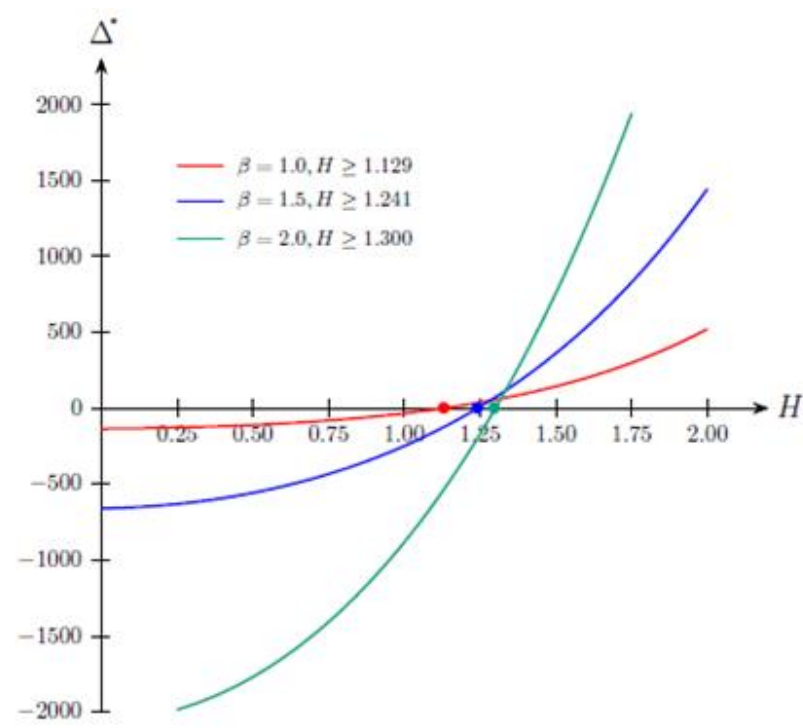

FIGURA 8: Valores de $\mathrm{H}$ a partir dos quais pode ocorrer bifurcação.

\subsection{PONTOS CRÍTICOS}

Detectam-se os pontos limites ao aplicar a condição $\frac{d \lambda^{p}}{d v}=0$ na trajetória primária de equilíbrio descrita na Equação 16. Este procedimento algébrico é descrito pela Equação 20.

Por outro lado, detectam-se os pontos de bifurcação impondo a condição de $u=0$ na Equação 18, o que leva às Equações 22.

Para calcular os fatores de carga limite $\lambda^{l}$ e de bifurcação $\lambda^{b}$, substituem-se os valores dados nas Equações 21 e 22, respectivamente, na Equação 16. Na Tabela 3 mostram-se os valores dos pontos críticos obtidos para $E=1, A_{0}=1, S=$ $2 H=3, \beta=1.0, \beta=1.5, \beta=2.0$.

$$
\begin{gathered}
\frac{\partial \lambda^{p}}{\partial v}=\left(6 \beta^{3} S^{3}+3 l_{0}^{3}\right) v^{2}+\left(12 H \beta^{3} S^{3}+6 \beta S l_{0}^{3}\right) v+\left(4 H^{2} \beta^{3} S^{3}+2 \beta^{2} S^{2} l_{0}^{3}\right)=0 \\
v_{1}^{l}=\frac{-\left(12 H \beta^{3} S^{3}+6 \beta S l_{0}^{3}\right)+\sqrt{\left(12 H \beta^{3} S^{3}+6 \beta S l_{0}^{3}\right)^{2}-\left(24 \beta^{3} S^{3}+12 l_{0}^{3}\right)\left(4 H^{2} \beta^{3} S^{3}+2 \beta^{2} S^{2} l_{0}^{3}\right)}}{\left(12 \beta^{3} S^{3}+6 l_{0}^{3}\right)} \\
v_{2}^{l}=\frac{-\left(12 H \beta^{3} S^{3}+6 \beta S l_{0}^{3}\right)-\sqrt{\left(12 H \beta^{3} S^{3}+6 \beta S l_{0}^{3}\right)^{2}-\left(24 \beta^{3} S^{3}+12 l_{0}^{3}\right)\left(4 H^{2} \beta^{3} S^{3}+2 \beta^{2} S^{2} l_{0}^{3}\right)}}{\left(12 \beta^{3} S^{3}+6 l_{0}^{3}\right)} \\
v_{1}{ }^{b}=\frac{-\beta S\left(2 H \beta^{2} S^{2}+l_{0}^{3}\right)+\beta S \sqrt{\left(2 H \beta^{2} S^{2}+l_{0}^{3}\right)^{2}-\beta S^{3}\left(2 \beta^{3} S^{3}+l_{0}^{3}\right)}}{\left(2 \beta^{3} S^{3}+l_{0}^{3}\right)} \\
v_{2}^{b}=\frac{-\beta S\left(2 H \beta^{2} S^{2}+l_{0}^{3}\right)-\beta S \sqrt{\left(2 H \beta^{2} S^{2}+l_{0}^{3}\right)^{2}-\beta S^{3}\left(2 \beta^{3} S^{3}+l_{0}^{3}\right)}}{\left(2 \beta^{3} S^{3}+l_{0}^{3}\right)}
\end{gathered}
$$

TABELA 3: Pontos críticos.

\begin{tabular}{ccccccccc}
\multicolumn{4}{c}{ Pontos limites } & \multicolumn{4}{c}{ Pontos de bifurcação } \\
\hline$\beta$ & $v_{1}{ }^{l}$ & $\lambda_{1}{ }^{l}$ & $v_{2}{ }^{l}$ & $\lambda_{2}{ }^{l}$ & $v_{1}{ }^{l}$ & $\lambda_{1}{ }^{l}$ & $v_{2}{ }^{l}$ & $\lambda_{2}{ }^{l}$ \\
\hline 1.0 & 1.0436 & 0.5044 & 3.6283 & -0.3082 & 0.1486 & 0.1446 & 4.5234 & 0.0516 \\
1.5 & 1.2679 & 0.5211 & 4.7321 & -0.5211 & 0.2182 & 0.1759 & 5.7818 & -0.1759 \\
2.0 & 1.3806 & 0.5136 & 5.0156 & -0.4335 & 0.2614 & 0.1890 & 6.1348 & -0.1089 \\
\hline
\end{tabular}

FONTE: Autoria Própria (2016). 

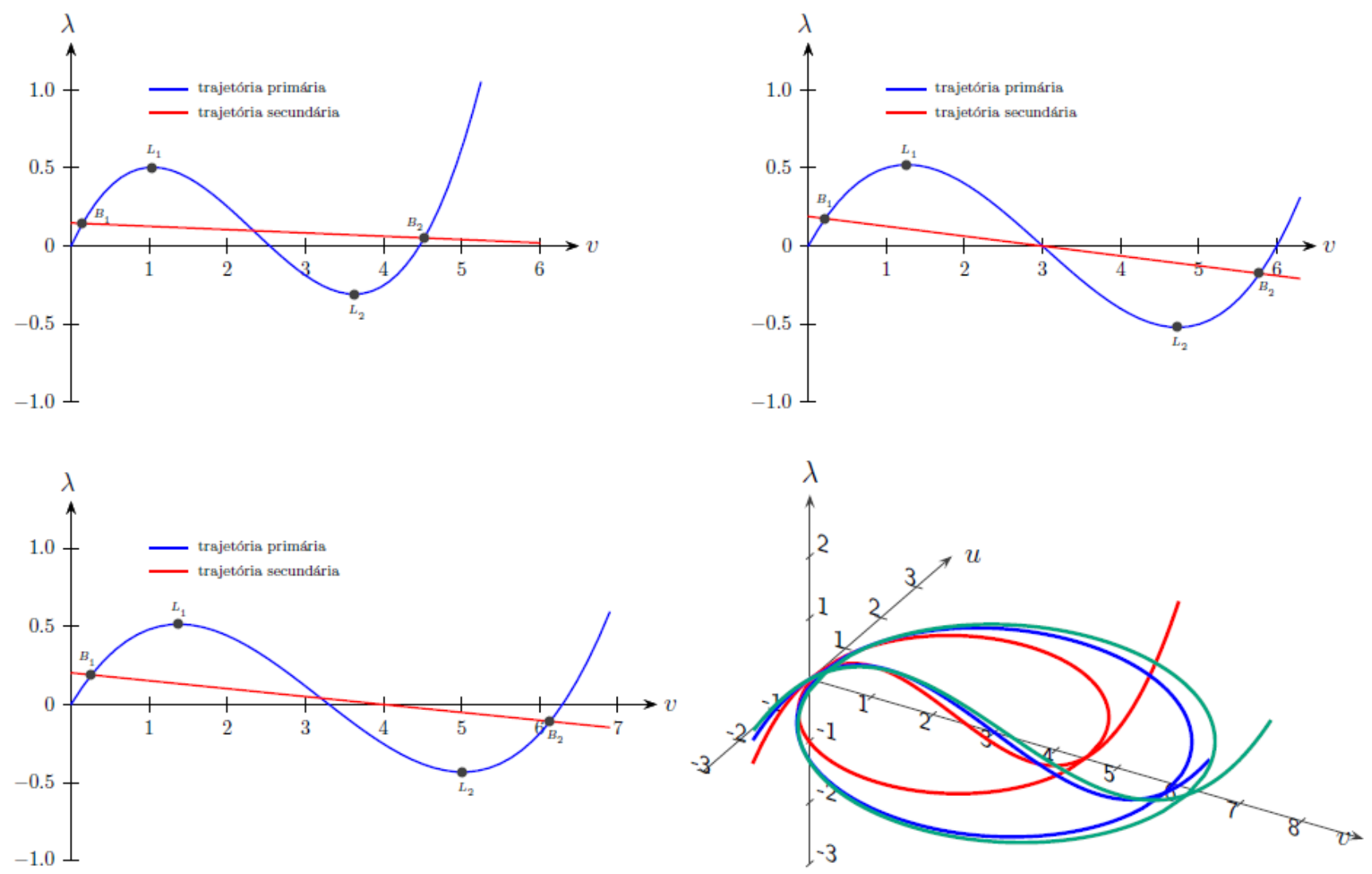

FIGURA 9: Trajetórias de equilíbrio. a) Trajetórias de equilíbrio para $\beta=1.0$. b) Trajetórias de equilíbrio para $\beta=1.5$. c) Trajetórias de equilíbrio para $\beta=2.0$. d) Trajetórias de equilíbrio no plano $(v, u, \lambda)$.

FONTE: Autoria Própria (2016).

\subsection{FATORES DE CARGA CRÍTICOS}

Como mostra-se nas Figuras 9a, 9b e 9c, as trajetórias de equilíbrio primárias possuem quatros pontos críticos, dois pontos limites, $L_{1}$ e $L_{2}$, e dois pontos de bifurcação, $B_{1}$ e $B_{2}$. Nessas figuras também observou-se que os primeiros pontos de bifurcação $B_{1}$ ocorreram antes dos primeiros pontos limites $L_{1}$ para os casos de $\beta=1.0, \beta=1.5$ e $\beta=2.0$. O propósito deste item é discutir a sequência de ocorrência desses pontos críticos. Para isso estudam-se as variações dos fatores de cargas para os primeiros pontos críticos em função das variações das propriedades geométricas da treliça. Aqui em particular propõe-se estudar a variação do fator de carga crítico em função da variação da altura da treliça, isto é, em função da variável $H$. Ao substituir o deslocamento vertical $v_{1}{ }^{l}$ dado pela Equação (21a) na equação (16) obtém-se o fator de carga para o primeiro ponto limite $\lambda_{1}{ }^{l}$. Este fator de carga estará em função das propriedades geométricas e mecânicas da treliça, isto é, $\lambda_{1}{ }^{l}\left(E, A_{0}, l_{0}, S, H, \beta\right)$. Por outro lado, ao substituir o deslocamento vertical $v_{1}^{b}$ dado pela Equação (22a) na Equação (16) obtém-se o fator de carga para o primeiro ponto de bifurcação $\lambda_{1}{ }^{b}$. Da mesma maneira, este fator de carga estará em função das propriedades geométricas e mecânicas da treliça, isto é, $\lambda_{1}{ }^{b}\left(E, A_{0}, l_{0}, S, H, \beta\right)$. Portanto para obter as curvas do fator de carga crítico em função da altura da treliça assumem-se os seguintes valores para as demais variáveis: $E=1, A_{0}=1, S=2$, e os valores $1,1.5$ e 2 para o parâmetro $\beta$. Nas Figuras $10 \mathrm{a}, 10 \mathrm{~b}$ e 10c mostram-se as curvas dos fatores de cargas críticos tanto para o ponto limite $\lambda^{p l}$ max quanto para o ponto de bifurcação $\lambda^{p b}{ }_{\max }$ para o intervalo $0<H<5$ e para os diferentes valores de $\beta$. Conforme mostra-se na Figura 10a para valores de $H<1.3458$ o ponto limite ocorre primeiro. Quando $H$ alcança o valor 1.3458 ocorre a coalescência entre o ponto limite e o ponto de 
bifurcação. Para $\mathrm{H}>1.3458$ o ponto de bifurcação ocorre primeiro. Ainda nesta figura observa-se que a carga de flambagem é muito menor que a carga limite e por fim mostra-se a capacidade portante máxima $\lambda_{\max }$ da treliça levando em conta a possibilidade de perda de rigidez da estrutura ou a flambagem. As mesmas observações podem ser feitas para as Figuras $10 \mathrm{~b}$ e 10c, com valores diferentes de $H$, em relação a sequência de ocorrência dos pontos críticos bem como da capacidade portante máxima da treliça em relação à perda de rigidez e à flambagem.

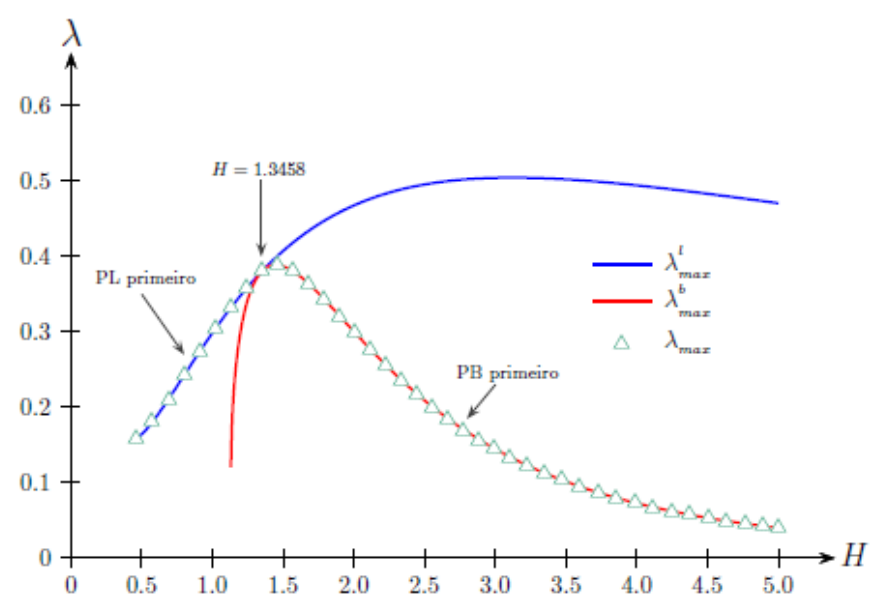

[a]

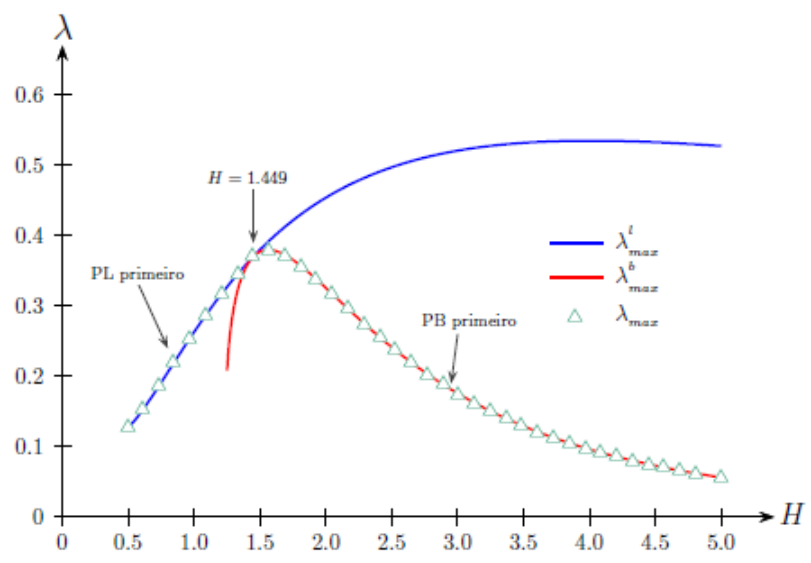

[b]

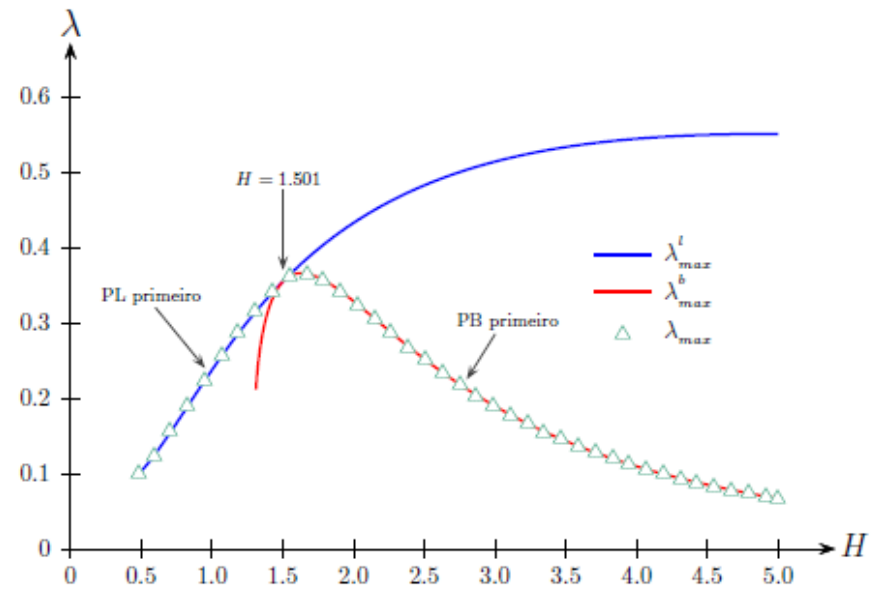

[c]

FIGURA 10: Fatores de carga críticos. [a] Carga crítica para $\beta=1.0$. [b] Carga crítica para $\beta=1.5$. [c] Carga crítica para $\beta=2.0$.

FONTE: Autoria Própria (2016). 


\section{CONLUSÕES}

De maneira sucinta pode-se dizer que neste trabalho foi descrito de forma objetiva, concisa e detalhada a abordagem analítica para a detecção, classificação e sequência de ocorrência de pontos críticos na trajetória primária de equilíbrio de um sistema físico simples. Determinou-se as condições geométricas e físicas para as quais ocorrem a coalescência entre os pontos limites e os pontos de bifurcação. A condição de coalescência de pontos críticos deve ser um requisito em estudos de otimização relativos à estabilidade estrutural. Portanto, é necessário levar em consideração a não linearidade geométrica na análise da estabilidade de equilíbrio de sistemas estruturais. É importante destacar é que o principal objetivo deste trabalho foi demonstrar a necessidade de compreender melhor os fenômenos não lineares para projetar sistemas estruturais mais seguros.

\section{REFERÊNCIAS BIBLIOGRÁFICAS}

BATHE, K.J., Finite element procedures. Prentice-Hall, 1037 p. 1996.

BELYTSCHKO T.; LIU W. K.; MORAN B. Nonlinear finite elements for continua and strucutures. John Wiley, 650 p. 2000.

BONET J.; WOOD R.D. Nonlinear continuum mechanics for finite element analysis. $2^{\text {nd }}$ Edition, Cambridge University Press, 318 p. 2008.

BORST R., CRISFIELD M.A., REMMERS J.J.C.; VERHOOSEL C.V. Non-linear finite element analysis of solids and structures. $2^{\text {nd }}$ Edition, Wiley, 516 p. 2012.

CRISFIELD M.A. Non-linear finite element analysis of solids and structures. Volume 1: Essentials. John Wiley, 345 p. 1991.

CRISFIELD M.A. Non-linear finite element analysis of solids and structures, Volume 2: Advanced Topics. John Wiley, 494 p. 1997.

DOYLE J.F. Nonlinear analysis of thin-walled structures. Statics, Dynamics and Stability. Springer, 511 p. 2001.

KRENK, S. Non-linear modeling and analysis of solids and structures. Cambridge University Press, 349 p. 2009.

WRIGGERS P. Nonlinear finite element methods, Springer, 559 p. 2008.
Wriggers P. Computational contact mechanics, 2nd Edition. Springer, 518 p. 2002. 